\title{
The Role of Macrolides in Unified Airway Disease (UAD) - A Review
}

\author{
Adam C Gaunt ${ }^{1^{*}}$, Rishi Sharma ${ }^{2}$ and Sally Erskine ${ }^{2}$ \\ ${ }^{1}$ West Suffolk Hospital, Bury St Edmunds, Suffolk, IP33 2QZ, UK \\ 2James Paget University Hospital, Gorleston, Great Yarmouth, NR31 6LA, UK
}

\begin{abstract}
Unified Airways Disease (UAD) encompasses distinct clinical entities including chronic rhinosinusitis and asthma and gives credence to the hypothesis that these are different facets of the same disease process. Macrolide antibiotics are derived from the prototypic macrolide erythromycin. This was discovered in the early 1950's as an isolate from the soil bacteria Saccharopolyspora erythraea and it is known to be a primarily bacteriostatic agent. Macrolides are a commonly used class of antibiotic that are known to have actions beyond their primary bactericidal functions and have been shown to be of benefit in conditions affecting all constituents of the airway, including chronic rhinosinusitis, asthma, diffuse panbronchiolitis and cystic fibrosis. These properties include potent anti-inflammatory and immunomodulatory effects. Promising results that have been shown with the use of macrolide therapies in airways diseases gives hope that there may be a wider application for them in Unified Airways Disease (UAD). A key property that macrolides (and newer generation ketolides) possess is the ability to interfere with protein translation at the 50s subunit of the bacterial ribosome. It is feasible that this action allows macrolides to disrupt the cellular processes related to bacterial proliferation and influence the inflammatory response, decreasing the production of inflammatory proteins and cytokines and disrupting biofilm formation. Macrolides are well established drugs with a known side-effect profile and relatively low cost and therefore could provide a cost-effective alternative to other costly therapies or surgeries.
\end{abstract}

Keywords: Unified airways disease; Macrolides; Chronic rhinosinusitis; Asthma; Chronic obstructive pulmonary disease; Cystic fibrosis

\section{The Unified Airway Hypothesis}

The concept of the Unified Airway (UA) has gained increased traction across the fields of respiratory medicine, allergy and otorhinolaryngology over the last 20 years [1]. It was first coined by Passalacqua et al. as United Airways Disease in 2000 [2], however seems to have given over to the term 'Unified Airway'. Understanding airways diseases as an interrelated entity could lead to improved understanding of these interdependent conditions and optimise treatment outcomes [3].

The central tenet of the unified airway is the concept of a contiguous tract, lined with respiratory epithelium encompassing the nose and middle ear and extending to the terminal bronchioles [4]. A pathological process in one part of this airway is in this way liable to affect the function of the rest of the airway, even at a site remote to the original insult [2].

Evidence for this hypothesis includes research that respiratory diseases such as asthma are linked to higher rates of disease in other parts of the airway, including rhinosinusitis $[5,6]$, and that there are also common histopathological hallmarks that are shared across clinically distinct entities such as asthma and chronic rhinosinusitis (CRS) [7].

\section{Chronic Rhinosinusitis (CRS) as a Component of UAD}

Nasal obstruction is a key component of CRS [8]. The nose serves to humidify and filter air and mechanical obstruction of the nasal airway may serve to thwart this. Mouth-breathing allows cold, unfiltered air to directly enter the bronchial tree and irritate the mucosa causing bronchospasm-this effect can be seen in exercise induced-asthma. A seminal study by Shturman-Ellstein et al. in 1978 demonstrated alterations in pulmonary physiology and changes in partial pressures of arterial oxygen in mouth-breathers, potentially due to changes in bronchial muscle reactivity [9]. Nasal obstruction may therefore also contribute to an alteration in the environment of the UA. The role of the nose and upper airway in the pathogenesis of respiratory disease is explained by four putative mechanisms $[10,11]$ :
1. Warming and humidification of inhaled air by the nasal mucosa and turbinates

2. Inflammatory products from the nose track into the lower airways from the upper airway

3. Nasal inflammation results in local cytokine release which is then absorbed systemically

4. Potential existence of a nasal-bronchial reflex via the afferent nasal sensory nerve

The observation that rates of asthma are much higher in those with CRS [6] is further evidence to strengthen the case for a unifying theory of airways disease. In a reciprocal manner, asthma symptoms are also shown to improve in patients who have undergone sinus surgery or medical treatment of their CRS $[12,13]$.

\section{The Role of Macrolides in Respiratory Disease}

Macrolides are a commonly-used class of antibiotics used in both acute and chronic respiratory infections and in those patients who have allergy to penicillin. A recent review article by Wong et al. gives a detailed examination of the role of macrolides in the management of asthma, stating that they have been shown to have 'antimicrobial, immunomodulatory and potential antiviral properties' [14]. They are mainstays in the management of chronic respiratory conditions such as Cystic Fibrosis and bronchiectasis.

The immunomodulatory characteristics that macrolides display

*Corresponding author: Adam Gaunt, West Suffolk Hospital, Bury St Edmunds, Suffolk, IP33 2QZ, George Borrow Road Norwich, Norfolk, NR4 7HU, UK, Tel: +44 7540 786158; E-mail: adamcgaunt@gmail.com

Received August 31, 2015; Accepted January 04, 2016; Published January 09 2016

Citation: Gaunt AC, Sharma R, Erskine S (2016) The Role of Macrolides in Unified Airway Disease (UAD) - A Review. J Pulm Respir Med 6: 312. doi:10.4172/2161105X.1000312

Copyright: $\odot 2016$ Gaunt AC, et al. This is an open-access article distributed under the terms of the Creative Commons Attribution License, which permits unrestricted use, distribution, and reproduction in any medium, provided the original author and source are credited. 
beyond their primary bactericidal properties [15] have become of increasing interest to those treating diseases of the respiratory tract. A key development was a report by a Japanese team in 1984 showed that there was a dramatic turn-around in the fortunes of patients they treated with diffuse panbronchiolitis (DPB) [16]. Patients with DPB traditionally had poor survival rates, however since the introduction of a low-dose erythromycin regime this has improved significantly, with clinical evidence that macrolides exert an anti-inflammatory response which includes the reduction of secretions and inflammatory mediators $[17,18]$.

\section{Asthma}

Although there is not strong enough evidence to support the widespread use of macrolides in asthma, certain specific subset of asthmatics may more responsive. Those with poorly controlled severe neutrophilic asthma [14] or those with Chlamydophila pneumoniae or Mycoplasma pneumonia positive PCR have derived benefit, demonstrating decreased airway hyperresponsiveness and increased peak flow in a trial of 6 weeks' Roxithromycin, although this was not sustained after cessation of the drug [19].

\section{Cystic fibrosis}

In Cystic Fibrosis (CF), trials have consistently shown benefit from the use of macrolides [20-22], although the mechanisms are not clear. Hypotheses include up-regulation of the multi-drug resistance (MDR) gene product P-Glycoprotein. This is thought improve function of the $\mathrm{CF}$ transporter receptor (CFTR) [23] and cause disruption of biofilms that are a characteristic finding in these patients. There is in-vitro evidence of a bactericidal effect that is mediated by prevention of adherence of bacteria to epithelial cells [24]. Adherence of bacteria to respiratory epithelium is known to be enhanced in CF due to thickened respiratory secretions and glycosylation of epithelial cells due to abnormalities in CFTR [20,24]. The repeat exacerbations and the progressive nature of the disease mean that a drug that can improve disease parameters is exciting, however this needs to be balanced against promotion of macrolide resistant strains $[20,25]$.

\section{Non-CF bronchiectasis}

Non-CF related bronchiectasis has historically lacked good evidence upon which to base treatment decisions [26]. Empirical treatment has been favoured with treatment regimes borrowed from its sister disease CF. This is despite there being some evidence that inhaled therapies such as Dornase alfa and Tobramycin have unintended negative effects on the frequency of exacerbations and lung function $[27,28]$. There is good evidence, at RCT and meta-analysis level that low dose azithromycin is of benefit in the reduction of severity and frequency of of exacerbations of bronchiectasis. The EMBRACE trial [29], using low dose Azithromycin showed a 38\% reduction in exacerbations and the BLESS trial [30] comparing low dose erythromycin noted $65 \%$ reduction. Meta-analysis by Wu et al. [31] shows a relative risk of 0.70 for exacerbations in those receiving long term macrolides vs placebo although this is balanced against significant increase in gastrointestinal upsets. An trend of increased microbial resistance was also noted, however analysis was not possible due to a lack of consistency between outcome measures.

\section{Chronic obstructive pulmonary disease}

Chronic obstructive pulmonary disease (COPD), is a disease with significant clinical burden. It characterised by irreversible airways obstruction and acute infective or inflammatory exacerbations and has been subjected to investigation regarding response to macrolide therapy. A large trial in $2011(\mathrm{n}=1142)$ by Albert et al. has shown a statistically significant benefit to quality of life in a subset of COPD patients who were given low dose azithromycin for one year in addition to their normal therapies. Acute exacerbations were reduced from 1.83 to 1.48 episodes per patient year in the treatment vs control group, although this resulted in some hearing decrements and colonisation with macrolide resistant species in some cases [32]. A 2013 Systematic review in the Cochrane database by Herath and Poole looked at 7 RCT's which used either continuous or pulsed prophylactic antibiotic regimes ( 5 vs 2). Only macrolides were used in the continuous regimens-namely azithromycin, erythromycin and clarithromycin. The authors found statistically significant reduction in the number of exacerbations, odds ratio 0.55 , number needed to treat avoid one exacerbation was 8 [33] An editorial July 2014 in American Journal of Respiratory and Critical Care Medicine makes the point that we are still far from justifying routine Azithromycin prophylaxis in COPD as several clinical questions remain unanswered-these are [34]:

1. Which subsets of patients will benefit most from Azithromycin?

2. How does Azithromycin fit in to the current COPD management guidelines?

3. There has not been a prospective RCT that 'takes into consideration all COPD pharmacological interventions and also provides guidelines on how to use corticosteroids and/or antibiotics during the exacerbations, we cannot conclude that using azithromycin prevents "more severe exacerbation."

\section{Mycobacterial infections}

Macrolides have previously been shown to be effective against nontuberculosis mycobacterium, however some varieties including M. abscessus and M. massiliense are known to develop rapid resistance in vitro [35]. M. tuberculosis is known to exhibit intrinsic macrolide resistance via the erm 37 gene product which prevents macrolide binding to the ribosome [36,37] - one of this class of antibiotics main weapons. Despite the lack of antimicrobial effect afforded by macrolides it is a possible that the immunomodulatory and synergistic effects alongside other antituberculous medicines may be useful in treating Mycobacterial infections. Clarithromycin has demonstrated a potentiation of the effects of rifampicin, isoniazid, ethambutol and pyrazinamide however this has not been validated in a clinical setting [38]. Newer forms of tuberculosis resistant to multiple drugs - so-called Multidrug Resistant Tuberculosis (MDRTB) have become prevalent and this is recognised to be a global health risk. Although macrolides need high mean inhibitory concentrations to be effective against TB, their propensity to collect in lung tissue, coupled with their immunomodulatory effects and synergistics could lead to their use as part of a regimen to treat MDRTB [39].

\section{Macrolides in CRS}

The current EPOS guidelines [8] remark that there only two placebo controlled studies examining the long-term use of macrolide antibiotics in CRS.

Wallwork et al. [40] looked specifically at patients with CRS without a history of nasal polyposis (CRSsNPs) using a regime of Roxithromycin $150 \mathrm{mg}$ daily for three months vs placebo and found that:

'There were statistically significant improvements in SNOT-20 score, nasal endoscopy, saccharine transit time, and IL-8 levels in lavage fluid $(P<0.05)$ in the macrolide group. A correlation was noted between improved outcome measures and low IgE levels. No significant 
improvements were noted for olfactory function, peak nasal inspiratory flow, or lavage levels for fucose and a2-macroglobulin'.

Videler et al. [41] compared a regime of three days' Azithromycin $500 \mathrm{mg}$, followed by weekly Azithromyin 500mg for three months. The findings were that:

'the SNOT-22,Patient Response Rating Scale, VAS scores and SF36, no significant difference between the AZM and the placebo groups was demonstrated. Nasal endoscopic findings, PNIF results, smell tests and microbiology showed no relevant significant differences between the groups either.al. looked at patients with both CRSsNPs and CRSwNPs.'

These conflicting answers and this may be due to the fact that one by Wallwork et al. looked only at CRSsNPs and whereas Videler et al. included patients with both CRSwNPs and CRSsNPs. The first study showed statistically significant improvements in subjective symptoms and biochemical and clinical indicators of disease, in comparison the second there were no significant benefits versus placebo. The fact that the two trials differed in patient selection may be a reason for this, therefore future trials should address this question, with two limbs identifying and stratifying these patients. A distinct subgroup existed in Wallwork's trial that had normal IgE levels these patients were identified as deriving particular benefit from macrolide use and further resources could be directed here. In light of this equivocal evidence, current CRS guidelines currently recommend that long-term antibiotics be used in CRS only where there is a positive bacterial culture and an acute exacerbation of symptoms.

\section{Macrolides-beyond simple antibiotics}

We have seen numerous examples of how macrolides have a beneficial effect in respiratory conditions that is not adequately explained in terms of bactericidal activity. The macrolides and their newer derivatives the ketolides are derived from the prototypic macrolide erythromycin. This was discovered in the early 1950's as an isolated from the soil bacteria Saccharopolyspora erythraea [42].

The chemical structure of erythromycin is a 14 -membered macrolactone ring, different members of the macrolide family being based upon this and the later 'ketolides' being characterised by the addition of a keto group. The mechanism of Erythromycin is primarily bacteriostatic rather than bactericidal and it displays instability in acidic conditions, this coupled with increasing bacterial resistance has led to the development of the semisynthetic macrolides such as clarithromycin and azithromycin and the ketolides, which are similar in structure but have superior stability and enhanced pharmacological properties [42].

A key property that macrolides (and newer generation ketolides) possess is the ability to interfere with protein translation at the $50 \mathrm{~s}$ subunit of the bacterial ribosome $[42,43]$. It is feasible that this action allows macrolides to disrupt the cellular processes related to bacterial proliferation and influence the inflammatory response. Effects upon white blood cell function, such as enhanced degranulation and chemotactic recruitment of neutrophils [15] as demonstrated in several in vitro and ex vivo studies [44] may further enhance their immunomodulatory properties. Extrapolating these observations from laboratory bench to the clinic is problematic however. Numerous cytokines (IL-6, 8, 10 and TNF) have also shown to be suppressed in a dose-dependent fashion by the administration of erythromycin in whole blood stimulated with Pseudomonas aeruginosa [45] in healthy subjects as well as in cell lines in vitro.

\section{Problems with macrolides}

The indiscriminate use of broad-spectrum antibiotics is known to promote colonisation with resistant organisms and the occurrence of iatrogenic infections such as C. difficile [46]. Any decision to commence long-term antibiotics at sub-bactericidal concentration therefore requires strong justification on clinical grounds. This is particularly true in conditions such as COPD and asthma where there is a large population of patients with relative immunocompromised. The risks of prescribing macrolides to a large cohort may have unintended harmful effects on the wider community due to promotion of resistant strains of bacteria, in particular with long-acting macrolides such as Azithromycin [47]. The side-effect profile of these drugs also needs to be considered as there are reports of significant cardiac events attributed to their use - patients should therefore be screened for evidence of QT segment prolongation or other cardiac abnormality before commencement [48]

The indiscriminate application of macrolides across the spectrum of UAD may be may mask the benefits they give to specific subgroups of patients with UAD. Greater benefit is likely to be derived from the targeted use of these drugs in specific groups of patients such as those with normal IgE levels in CRS or neutrophil mediated asthma [14]. This must however be balanced against the danger of promoting macrolide resistance in patients who are in some cases already at risk of infection, especially if these are used as a monotherapy and for long courses at sub-bactericidal doses $[20,23,25,42]$. In addition, it is yet to be determined if the immunomodulatory effects give benefit above and beyond traditional management strategies in more than a few select scenarios (e.g. DPB).

\section{Conclusion}

There is a growing body of evidence that supports the use of macrolides in UAD. Although there are common themes that run through the diseases of the airways they remain a heterogeneous group of diseases.

The action of macrolides beyond their antibacterial function is not fully understood, however it is thought to be related to a potent antiinflammatory action and immunomodulatory properties. Macrolides influence protein transcription at the ribosome [42], inhibiting the expression of inflammatory cytokines and therefore reduce inflammation. In cystic fibrosis models they are able to disrupt bacterial adhesion to epithelial cells as well as interfering with the formation of biofilms [15].

The varied mechanisms of action that macrolides possess make them an attractive treatment option as they have the potential to modify airways disease and reduce their significant socio-economic burden. Greater understanding of their beneficial actions may also lead to novel agents to combat UAD. Macrolides are well established drugs with a known side-effect profile and low cost and therefore could provide a cost-effective alternative to other costly therapies or surgeries.

New studies to examine these effects may benefit from accurately identifying subgroups in order to ensure that benefits in these groups are not overlooked in the analysis. Robust outcome measures that encompass both quality of life indices and hard outcomes such as hospital admissions or 5 year survival will also help to determine any benefit from these drugs. There may be great benefit to be derived from the macrolides for some patient groups but this will only be identified with careful study design.

\section{References}

1. Krouse JH (2012) The unified airway. Facial Plast Surg Clin North Am 20: 55- 
Citation: Gaunt AC, Sharma R, Erskine S (2016) The Role of Macrolides in Unified Airway Disease (UAD) - A Review. J Pulm Respir Med 6: 312. doi:10.4172/2161105X.1000312

60.

2. Passalacqua G, Ciprandi G, Canonica GW (2000) United airways disease: therapeutic aspects. Thorax 55 Suppl 2: S26-27.

3. Brozek JL, Bousquet J, Baena-Cagnani CE, Bonini S, Canonica GW, et al (2010) Allergic Rhinitis and its Impact on Asthma (ARIA) guidelines: 2010 revision. J Allergy Clin Immunol 126: 466-476.

4. Krouse JH (2008) The unified airway--conceptual framework. Otolaryngol Clin North Am 41: 257-266, v.

5. Lipworth BJ, White PS (2000) Allergic inflammation in the unified airway: start with the nose. Thorax 55: 878-881.

6. Jani AL, Hamilos DL (2005) Current thinking on the relationship between rhinosinusitis and asthma. J Asthma 42: 1-7.

7. Bachert C, Vignola AM, Gevaert P, Leynaert B, Van Cauwenberge P, et al (2004) Allergic rhinitis, rhinosinusitis, and asthma: one airway disease. Immuno Allergy Clin North Am 24: 19-43.

8. Fokkens WJ, Lund VJ, Mullol J, Bachert C, Alobid I, et al. (2012) EPOS 2012 European position paper on rhinosinusitis and nasal polyps 2012. A summary for otorhinolaryngologists. Rhinology 50: 1-12.

9. Shturman-Ellstein R, Zeballos RJ, Buckley JM, Souhrada JF (1978) The beneficial effect of nasal breathing on exercise-induced bronchoconstriction. Am Rev Respir Dis 118: 65-73.

10. Feng $\mathrm{CH}$, Miller MD, Simon RA (2012) The united allergic airway: connections between allergic rhinitis, asthma, and chronic sinusitis. Am J Rhinol Allergy 26 187-190.

11. Togias A (1999) Mechanisms of nose-lung interaction. Allergy 54 Suppl 57: 94 105

12. Zhang Z, Adappa ND, Doghramji LJ, Chiu AG, Lautenbach E, et al. (2014) Quality of life improvement from sinus surgery in chronic rhinosinusitis patients with asthma and nasal polyps. Int Forum Allergy Rhinol 4: 885-892.

13. Passalacqua G, Ciprandi G, Canonica GW (2001) The nose-lung interaction in allergic rhinitis and asthma: united airways disease. Curr Opin Allergy Clin Immunol 1: 7-13.

14. Wong EH, Porter JD, Edwards MR, Johnston SL (2014) The role of macrolides in asthma: current evidence and future directions. Lancet Respir Med 2: 657670.

15. Schultz MJ (2004) Macrolide activities beyond their antimicrobial effects: macrolides in diffuse panbronchiolitis and cystic fibrosis. J Antimicrob Chemother 54: 21-28.

16. Kudoh S, Azuma A, Yamamoto M, Izumi T, Ando M (1998) Improvement of survival in patients with diffuse panbronchiolitis treated with low-dose erythromycin. Am J Respir Crit Care Med 157: 1829-1832.

17. Goswami SK, Kivity S, Marom Z (1990) Erythromycin inhibits respiratory glycoconjugate secretion from human airways in vitro. Am Rev Respir Dis 141: 72-78

18. Tamaoki J, Isono K, Sakai N, Kanemura T, Konno K (1992) Erythromycin inhibits Cl secretion across canine tracheal epithelial cells. Eur Respir J 5: 234-238.

19. Black PN, Blasi F, Jenkins CR, Scicchitano R, Mills GD, et al. (2001) Trial of roxithromycin in subjects with asthma and serological evidence of infection with Chlamydia pneumoniae. Am J Respir Crit Care Med 164: 536-541.

20. Equi AC, Davies JC, Painter H, Hyde S, Bush A, et al. (2006) Exploring the mechanisms of macrolides in cystic fibrosis. Respir Med 100: 687-697.

21. Equi A, Balfour-Lynn IM, Bush A, Rosenthal M (2002) Long term azithromycin in children with cystic fibrosis: a randomised, placebo-controlled crossover trial. Lancet 360: $978-984$

22. Wolter J, Seeney S, Bell S, Bowler S, Masel P, et al. (2002) Effect of long term treatment with azithromycin on disease parameters in cystic fibrosis: a randomised trial. Thorax 57: 212-216.

23. Hyde SC, Emsley P, Hartshorn MJ, Mimmack MM, Gileadi U, Pearce SR, et al. (1990) Structural model of ATP-binding proteins associated with cystic fibrosis, multidrug resistance and bacterial transport. Nature 346: 362-365.

24. Saiman L, Prince A (1993) Pseudomonas aeruginosa pili bind to asialoGM1 which is increased on the surface of cystic fibrosis epithelial cells. J Clin Invest 92: 1875-1880.

25. Simoens S, Laekeman G, Decramer M (2013) Preventing COPD exacerbations with macrolides: a review and budget impact analysis. Respir Med 107: 637-648.
26. Serisier DJ (2014) The evidence base for non-CF bronchiectasis is finally evolving. Respirology 19: 295-297.

27. Barker AF, Couch L, Fiel SB, Gotfried MH, llowite J, et al. (2000) Tobramycin solution for inhalation reduces sputum Pseudomonas aeruginosa density in bronchiectasis. Am J Respir Crit Care Med 162: 481-485.

28. O'Donnell AE, Barker AF, Ilowite JS, Fick RB (1998) Treatment of idiopathic bronchiectasis with aerosolized recombinant human DNase I. rhDNase Study Group. Chest 113: 1329-1334.

29. Wong C, Jayaram L, Karalus N, Eaton T, Tong C, et al. (2012) Azithromycin for prevention of exacerbations in non-cystic fibrosis bronchiectasis (EMBRACE): a randomised, double-blind, placebo-controlled trial. Lancet 380: 660-667.

30. Serisier DJ, Martin ML (2011) Long-term, low-dose erythromycin in bronchiectasis subjects with frequent infective exacerbations. Respir Med 105: 946-949.

31. Wu Q, Shen W, Cheng H, Zhou X (2014) Long-term macrolides for non-cystic fibrosis bronchiectasis: a systematic review and meta-analysis. Respirol Carlton Vic 19: 321-329.

32. Albert RK, Connett J, Bailey WC, Casaburi R, Cooper JA Jr, et al. (2011) Azithromycin for prevention of exacerbations of COPD. N Engl J Med 365 689-698.

33. Herath SC, Poole P (2013) Prophylactic antibiotic therapy for chronic obstructive pulmonary disease (COPD). Cochrane Database Syst Rev 11: CD009764.

34. Restrepo MI, Anzueto A (2014) Macrolide antibiotics for prevention of chronic obstructive pulmonary disease exacerbations: are we there yet? Am J Respir Crit Care Med 190: 1-2

35. Kim SY, Kim CK, Bae IK, Jeong SH, Yim JJ, et al. (2015) The drug susceptibility profile and inducible resistance to macrolides of Mycobacterium abscessus and Mycobacterium massiliense in Korea. Diagn Microbiol Infect Dis 81: 107-111.

36. Andini N, Nash KA (2006) Intrinsic macrolide resistance of the Mycobacterium tuberculosis complex is inducible. Antimicrob Agents Chemother 50: 2560-2562.

37. Dooley KE, Obuku EA, Durakovic N, Belitsky V, Mitnick C, et al. (2013) World Health Organization group 5 drugs for the treatment of drug-resistant tuberculosis: unclear efficacy or untapped potential? J Infect Dis 207: 1352-1358.

38. Cavalieri SJ, Biehle JR, Sanders WE (1995) Synergistic activities of clarithromycin and antituberculous drugs against multidrug-resistant Mycobacterium tuberculosis. Antimicrob Agents Chemother 39: 1542-1545

39. van der Paardt AF, Wilffert B, Akkerman OW, de Lange WC, van Soolingen D, et al. (2015) Evaluation of macrolides for possible use against multidrug-resistant Mycobacterium tuberculosis. Eur Respir J 46: 444-455.

40. Wallwork B, Coman W, Mackay-Sim A, Greiff L, Cervin A (2006) A Double-Blind Randomized, Placebo-Controlled Trial of Macrolide in the Treatment of Chronic Rhinosinusitis. Laryngoscope 116: 189-193.

41. Videler WJ, Badia L, Harvey RJ, Gane S, Georgalas C, et al. (2011) Lack of efficacy of long-term, low-dose azithromycin in chronic rhinosinusitis: a randomized controlled trial. Allergy 66:1457-1468.

42. Douthwaite S, Champney WS (2001) Structures of ketolides and macrolides determine their mode of interaction with the ribosomal target site. J Antimicrob Chemother 48 Suppl T1: 1-8.

43. Mazzei T, Mini E, Novelli A, Periti P (1993) Chemistry and mode of action of macrolides. J Antimicrob Chemother 31 Suppl C: 1-9.

44. Adams DH, Lloyd AR (1997) Chemokines: leucocyte recruitment and activation cytokines. Lancet 349: 490-495.

45. Schultz MJ, Speelman P, Hack CE, Buurman WA, van Deventer SJH, et al. (2000) Intravenous infusion of erythromycin inhibits CXC chemokine production, but augments neutrophil degranulation in whole blood stimulated with Streptococcus pneumoniae. J Antimicrob Chemother 46: 235-240.

46. Goossens H, Ferech M, Vander Stichele R, Elseviers M; ESAC Project Group (2005) Outpatient antibiotic use in Europe and association with resistance: a cross-national database study. Lancet 365: 579-587.

47. Serisier DJ (2013) Risks of population antimicrobial resistance associated with chronic macrolide use for inflammatory airway diseases. Lancet Respir Med 1: $262-274$.

48. Owens RC Jr, Nolin TD (2006) Antimicrobial-associated QT interval prolongation: pointes of interest. Clin Infect Dis 43: 1603-1611. 